\title{
Ultrasound-assisted transdermal delivery of alendronate for the treatment of osteoporosis
}

\author{
Baofeng Li', Guiyun Huang ${ }^{2}$, Zhenhe $\mathrm{Ma}^{3}$ and Shourong Qin ${ }^{4 ⿴ 囗}$ \\ 1Department of Minimally Invasive Orthopaedics, Shandong Provincial Hospital of Traditional Chinese Medicine, Jinan, Shandong Province, \\ 250011, China; 2Department of Orthopaedics, Jiyang Public Hospital of Shandong Province, Jinan, Shandong Province, 251400, China; ${ }^{3}$ Depart- \\ ment of Rehabilitation, Ningjin County Hospital of Traditional Chinese Medicine, Dezhou, Shandong Province, 253400, China; ${ }^{4 *}$ Department of \\ Traumatic Orthopaedics, Taian City Central Hospital, No.29, Longtan Road, Taian, Shandong Province, 271000, China
}

The aim of the current research work was to develop sonophoresis-assisted transdermal patches for the treatment of osteoporosis. In the present investigation, we formulated alendronate-chitosan nanoparticles by ionotropic external gelation method. The prepared nanoparticles were found to be smooth and free-flowing. The optimized formulation showed $82.7 \%$ of drug release over a period of 12 hours with $99.54 \% \mathrm{EE}$, the particle size of $250 \mathrm{~nm}$, PDI 0.22 and zeta potential of $28 \mathrm{mV}$. The solvent casting evaporation method was used for the development of the patches using HPMC as rate-controlling polymer and dibutyl phthalate as the plasticizer. The optimized patch formulation was found acceptable in terms of physical characteristics (appearance, thickness, folding endurance, weight variation, moisture loss and uptake). The drug content was found to be $99.66 \pm 0.9 \%$ with $69.44 \%$ of drug permeation through the rat skin. The TP3 formulation had drug content of $99.96 \%$ which was the highest among all of the formulations and showed relatively controlled skin permeation of $69.44 \%$ over the period of 12 hours. Nearly six-time enhancement of bioavailability was observed when alendronate was used in the nanoparticulate form in transdermal patches used with sonophoresis. Over the period of seven days, the plasma calcium concentration in the rat model was decreased from $16 \mathrm{mg} / \mathrm{dl}$ to $4 \mathrm{mg} / \mathrm{dl}$ (4 times) in rat groups treated with the transdermal patches containing CS-ALN-NP while the concentration dropped only to $12 \mathrm{mg} / \mathrm{dl}$ in case of the transdermal patches containing pure Alendronate. These findings (enhanced skin permeation, enhanced bioavailability and suppression of the plasma calcium level) regarding the transdermal delivery system suggest a promising approach for the treatment of osteoporosis.

Key words: sonophoresis, alendronate nanoparticles, rat, osteoporosis, transdermal patch

Received: 13 January, 2020; revised: 16 April, 2020; accepted: 04 May, 2020; available on-line: 19 June, 2020

घe-mail: shourongqin@sina.com

Abbreviations: ALN, Alendronate; CS, chitosan with $80 \%$ deacetylation; DLS, dynamic light scattering; HPMC, hydroxypropyl methylcellulose; PVA, polyvinyl alcohols; S.E.M., scanning electron microscopy; TPP, tripolyphosphate

\section{INTRODUCTION}

Osteoporosis is a common chronic metabolic bone disease, related to various factors including menopause and aging and it is characterized by increased bone fragility. Osteoporosis involves a disruption of bone microarchitecture with low bone mass and deterioration of the bone tissue. It all it can lead to weakened bone strength and an increase in the risk of bone fractures. Osteoporosis causes more than 8 million fractures per year which makes it a worldwide concern (Sözen et al., 2017; Johnell et al., 2006). Bisphosphonates, like alendronate, show a high affinity for hydroxyapatite which results in prevention of the bone resorption, and thus are widely used for the prevention and treatment of various bone diseases, including hypercalcemia due to malignancy, Paget's disease and osteoporosis (Schenk et al., 1986; Fleisch., 1987; Lambrinoudaki et al., 2006; VanBeek et al., 2003). Bisphosphonates are mainly classified into two groups depending on their different molecular modes of action (Ezra et al., 2000; Drake et al., 2008). The first class are bisphosphonates without nitrogen moiety, like clodronate and etidronate, which can be metabolically incorporated into adenosine triphosphate analogues that may inhibit ATP-dependent intracellular enzymes (Fleisch, 2002; Russell, 2007). The second class are Bisphosphonates with nitrogen moiety like pamidronate, alendronate, risedronate, ibandronate, and zoledronate. These are more potent and they inhibit the enzymes of the mevalonate pathway responsible for the biosynthesis of isoprenoid compounds essential for the posttranslational modification of small GTP-binding proteins (Fisher et al., 2000). The inhibition of protein prenylation and the disturbance in the function of these key regulatory proteins explain the loss of osteoclast activity and induction of apoptosis (Rodan et al., 2002). Among the bisphosphonates with nitrogen moiety, alendronate sodium (sodium-4-amino1-hydroxybutylidene-1,1-bisphosphonate trihydrate) has been not only used as the first-choice drug for the treatment of hypercalcemia and osteoporosis in postmenopausal women but also for the therapy of bone diseases such as Paget's disease (Dunford et al., 2006). However, alendronate oral bioavailability is approximately $0.9 \%$ to 1.8\% (Rodan et al., 2002). In addition, the administration of alendronate via oral route results in mucosal damage, including gastric ulcer, gastritis and erosive esophagitis. To avoid these adverse effects of alendronate, patients after oral administration need to sit up or walk for more than 30 minutes. More than $70 \%$ of osteoporosis patients are elderly people, most of them have difficulties following the protocol associated with oral administration of alendronate, leading to poor compliance and quality of life in these patients. 
Sonophoresis or phonophoresis has been widely used to deliver therapeutic molecules through the skin. This technique involves the use of ultrasound of high frequency or low frequency to increase the soft tissue or skin permeation of the drugs. This technique has been widely used since 1950 as it is painless and easy to handle and enables an easy transfer of low molecular weight drugs as well as macromolecules across the skin. It enhances the skin permeation by thermal, mechanical, radiation pressure and convection effect and by increasing the solution membrane interfacial transfer rate (Morimoto et al., 2005; Paliwal et al., 2006). Among the various strategies available for the administration of alendronate, the transdermal delivery may improve the compliance and quality of life of patients because of its advantages like ease of use for elderly and bedridden patients, and the avoidance of intestinal damage (Reszka et al., 2003). However, due to high polarity and hydrophilicity alendronate may not be readily absorbed through the skin. The layers of lipids of the stratum corneum in the skin limit the transdermal absorption of highly polar and hydrophilic chemicals. Considering the potential of sonophoresis as an efficient tool for enhancing the permeation for drug molecules we attempted to enhance the skin permeation of alendronate in the treatment of osteoporosis. Although some transdermal patches that overcome this issue have been already developed, alendronate was precipitated in the conventional lipophilic adhesives used in the preparation of these patches (Sato et al., 1990). The aim of the present study was to develop a novel transdermal drug delivery system utilizing sonophoresis for the treatment of osteoporosis.

\section{MATERIAL AND METHODS}

Material. Alendronate (ALN) was supplied by Baoji Guokang Bio-Technology Co., Ltd. (Baoji, Shaanxi, China); chitosan with $80 \%$ deacetylation (CS), tripolyphosphate (TPP), Tween-80, hydroxypropyl methylcellulose (HPMC), polyvinyl alcohols (PVA), dibutyl phtalate, acetic acid, methanol were of analytical grade purchased from Shanghai Chemical Co. (Shanghai, China).

Method of preparation. Alendronate-loaded chitosan nanoparticles. Chitosan-alendronate nanoparticles were prepared using the ionotropic external gelation method (Wen et al., 2009). Chitosan was dissolved in acetic acid under stirring at room temperature to get a clear solution. Tween-80 was added as a surfactant. Alendronate dissolved in acetone was added dropwise to the aqueous phase of the chitosan solution. Tripolyphosphate was added dropwise at different concentration into an $\mathrm{o} / \mathrm{w}$ emulsion under stirring as the cross-linking agent. For complete evaporation of the organic solvent, the formulation was kept overnight. The interaction between negative groups of tripolyphosphate and positively charged amino groups of chitosan resulted in nanoparticles formation. By using cooling centrifuge nanoparticles were separated by centrifugation at $20000 \mathrm{rpm}$ for 15 minutes at $-80^{\circ} \mathrm{C}$, and the supernatant was used for the measurement of free alendronate by HPLC. The glass bottles containing the samples were filled with chitosanalendronate nanoparticulate suspension by adding trehalose as a cryoprotectant and placed in cooling trap assembly of the instrument for $10 \mathrm{~h}$ at $-60^{\circ} \mathrm{C}$. During freezing the temperature varied from $15^{\circ} \mathrm{C}$ to $-40^{\circ} \mathrm{C}$ to get free-flowing powder of nanoparticles. The prepared alendronate-loaded chitosan nanoparticles were characterized and optimized nanoparticles were used for the
Table 1. Formulation batches of chitosan-alendronate nanoparticles.

\begin{tabular}{llllll}
\hline Ingredients & F1 & F2 & F3 & F4 & F5 \\
\hline Alendronate $(\mathrm{mg})$ & 100 & 100 & 100 & 100 & 100 \\
\hline Acetone $(\mathrm{ml})$ & 10 & 10 & 10 & 10 & 10 \\
\hline Chitosan $(\mathrm{mg})$ & 50 & 100 & 200 & 300 & 400 \\
\hline Acetic acid $(\mathrm{ml})$ & 3 & 3 & 3 & 3 & 3 \\
\hline TPP $(\mathrm{mg})$ & 10 & 20 & 30 & 40 & 50 \\
\hline Tween-80 $(\mathrm{ml})$ & 5.0 & 7.5 & 10.0 & 12.5 & 15.0 \\
\hline Purified water $(\mathrm{ml})$ & 100 & 100 & 100 & 100 & 100 \\
\hline
\end{tabular}

preparation of alendronate-loaded nanoparticulate patches. Formula composition is presented in Table 1.

Formulation of the patches by solvent casting evaporation method. Transdermal patches of chitosanalendronate nanoparticles were prepared by the solvent casting-evaporation technique in a glass petri dish (Allemann et al., 1993). The polyvinyl alcohol was dissolved in double distilled water by heating the solution to $40^{\circ} \mathrm{C}$. The baking membrane was cast by pouring polyvinyl alcohol solution into a glass petri dish followed by drying in a hot-air oven at $55^{\circ} \mathrm{C}$ for 8 hours. The drug reservoir compartment was prepared by dissolving hydroxyl propyl methyl cellulose in double-distilled water. To this solution dibutyl phtalate as a plasticizer was added. Optimized chitosan-alendronate nanoparticles were suspended in methanol and then added drop by drop into the hydroxylpropyl methylcellulose-plasticizer dispersion under slow stirring with a magnetic stirrer. This whole dispersion containing drug was cast onto the polyvinyl alcohol backing membrane and kept for drying for 6 hours at $50^{\circ} \mathrm{C}$. After completely drying, the patch was removed from the petri dish, cut into small patches and kept in a desiccator for further analysis. Formula composition of the transdermal patches is presented in Table 2.

\section{1) $\%$ encapsulation efficiency}

The percentage of encapsulation efficiency of CSALN-NP was determined by the indirect method (Rodan et al., 2003). CS-ALN-NP were separated from the suspension by ultracentrifugation for $30 \mathrm{~min}$ at $15000 \mathrm{rpm}$, $4^{\circ} \mathrm{C}$. The amount of free alendronate or non-encapsulated alendronate in the supernatant was analyzed by HPLC (Waters, Germany). The supernatant solution (25 microliters) was injected into a chromatograph equipped with a C18 column and UV detector. The flow rate of the mobile phase ( $90 \%$ acetonitrile: 10\% HPLC grade water $+0.35 \%$ triethylamine $+0.15 \%$ ortho-phosphoric

Table 2. Formulation batches of the transdermal patches containing CS-ALN-NP.

\begin{tabular}{lccccc}
\hline Ingredients & TP1 & TP2 & TP3 & TP4 & TP5 \\
\hline $\begin{array}{l}\text { Chitosan-alendronate } \\
\text { NP eq. to (mg) }\end{array}$ & 100 & 100 & 100 & 100 & 100 \\
& & & & & \\
\hline Polyvinyl alcohol (mg) & 200 & 300 & 400 & 450 & 500 \\
\hline HPMC low-viscosity (mg) & 5 & 10 & 15 & 20 & 25 \\
\hline Dibutyl pthalate (ml) & 0.2 & 0.3 & 0.4 & 0.5 & 0.6 \\
\hline Purified water (ml) & 100 & 100 & 100 & 100 & 100 \\
\hline
\end{tabular}

Characterization of the nanoparticles: 
acid) was $3 \mathrm{ml} / \mathrm{min}$; the wavelength was set to $260 \mathrm{~nm}$. The percentage of encapsulation efficiency (E.E) of the nanoparticles was calculated according to the following equations.

$\%$ E.E $=$ Total drug - Free drug $/$ Total drug amount x 100

\section{2) Particle size, PDI (Polydispersity index)}

The size of CS-ALN-NP was determined using a laser diffraction particle size analyzer at room temperature. The CS-ALN-NP were dissolved in Millipore pure water and then sonicated in an ultrasonicator for $30 \mathrm{~min}$. Then, the polydispersity index was determined by dynamic light scattering (DLS) using a ZetaNano ZS instrument (Dolatabadi et al., 2014).

\section{3) Zeta potential}

Zeta potential of CS-ALN-NP was determined by dynamic light scattering (DLS) using a Zeta Nano ZS instrument (Reid, 2014).

\section{4) In vitro drug release}

The release of alendronate from CS-ALN-NP was determined by dialysis bag/membrane (Allemann et al., 1993) (molecular cut-off of $5 \mathrm{kDa}$ ) method. $50 \mathrm{mg}$ equivalent CS-ALN-NP was dispersed in $5 \mathrm{ml}$ of phosphate buffer of $\mathrm{pH} 6.8$ in dialysis tube tied at both ends. The tube was placed in a beaker containing $900 \mathrm{ml}$ of buffer solution. The system was maintained at $37^{\circ} \mathrm{C}$ and stirred with a magnetic stirrer. $5 \mathrm{ml}$ of the solution was removed at predetermined time points and replenished with fresh solution to maintain the sink condition. Alendronate release at each time point was measured by the HPLC method as described above.

\section{5) SEM}

The shape and surface characteristics of the CS-ALNNP were observed by scanning electron microscopy (S.E.M.).

\section{Development of transdermal patches containing CS-ALN-NP}

Characterization of chitosan-alendronate transdermal patches (Kaveh et al., 2010).

Physical appearance

All the prepared chitosan-alendronate patches were visually inspected and examined for color, smoothness, clarity, and flexibility.

\section{Thickness}

By using digital micrometer screw gauge the thickness of chitosan-alendronate prepared patches were measured at 3 different locations and the thickness was determined by calculating the average of the 3 values.

Weight variation:

By using digital electronic balance the weight variation test was performed: 10 patches from each batch were weighed and the average weight and standard deviation values were calculated.

\section{Folding endurance}

The total number of folds required to break a polymeric patch is called folding endurance. The strength of the prepared chitosan-alendronate patches and efficiency of the plasticizer was determined by performing the folding endurance test. To perform this test a 2 $\mathrm{cm} \times 2 \mathrm{~cm}$ strip of the patch was cut evenly and repeat- edly folded at the same place until it broke. The total number of times the patch could be folded at the same place without breaking or cracking was the value of folding endurance.

\section{Drug content uniformity of patches}

Drug content uniformity of patches was assessed on three chitosan-alendronate patches. Each patch was dissolved in $50 \mathrm{ml}$ of methanol and the aliquots were separated from non-dissolved residues by filtration. The drug content was determined by measuring spectrophotometrically at $260 \mathrm{~nm}$.

\section{Tensile strength}

The tensile strength of the patch was determined for characterization of its mechanical properties by using the universal strength testing apparatus (Hounsfield, Slinfold, Horsham, UK)

\section{Percentage moisture absorption}

The integrity of patches at high humidity conditions was assessed by measuring the percent moisture absorption by the patches. The individual patches were weighed and then were kept in desiccators containing a saturated solution of potassium chloride at room temperature for 24 hours. Then, the patches were reweighed and the percent moisture absorption was determined by using the following formula:

Percent moisture absorption = Final weight - Initial weight $/$ Initial weight $\times 100$

\section{Percentage moisture loss}

Percent moisture loss was generally used to determine the stability of the prepared patches under ambient humidity conditions. Patches with very little moisture content become brittle. Percent moisture loss was determined by pre-weighting of patches, keeping them in desiccators containing silica for three days and weighting them again, and the percent moisture loss was determined using the following formula:

Percent moisture loss $=$ Initial weight - Final weight $/$ Final weight $\times 100$

In vitro skin permeation study using ultrasonically-treated rat skin (Mccarthy et al., 2002)

The rat abdominal skin was obtained as follows: the rats were kept under pentobarbital anesthesia $(40 \mathrm{mg}$ / $\mathrm{kg}$ ), the hair on the skin was removed using an animal hair clipper and full-thickness skin was excised. The excess fat adhering to the dermis side was removed using cotton. All animal experiments were done as per institutional ethical committee guidelines and approval. The rat abdominal skin was treated with low-frequency ultrasonic waves of $20 \mathrm{kHz}$ (VCX 130; Sonics and Materials, Newton, CT, USA). Such ultrasound-treated skin with a diameter of $20 \mathrm{~mm}$ was mounted between the receptor and donor chambers. The skin was mounted in such a way that the stratum corneum faced the donor compartment. The patch was applied to the stratum corneum side of the skin. The receptor compartment of the cell was filled with $3 \mathrm{ml}$ of phosphate buffer solution $\mathrm{pH}$ 7.4. The constant temperature of $32^{\circ} \mathrm{C}$ was maintained in the cells. $0.3 \mathrm{ml}$ of the solution from the receptor chamber was withdrawn periodically and replaced with an equal volume of fresh phosphate buffer solution. The alendronate concentration in each receptor chamber 
Table 3. Characterization of CS-ALN-NP

\begin{tabular}{llllll}
\hline Parameter & F1 & F2 & F3 & F4 & F5 \\
\hline E.E (\%) & 80.2 & 85.9 & 92.17 & 95.18 & 99.54 \\
\hdashline Particle size $(\mathrm{nm})$ & 190 & 205 & 210 & 220 & 250 \\
\hdashline PDI & 0.49 & 0.39 & 0.45 & 0.5 & 0.22 \\
\hdashline Zeta Potential (mV) & 18 & -30 & 20 & 22 & 28 \\
\hline
\end{tabular}

sample was analyzed using the reverse-phase high-performance liquid chromatography (RP-HPLC) method.

Hypercalcemia experiment (Haddad; 1998)

To study the hypercalcemia effect and to establish a hypercalcemia-like state $2.5 \mathrm{mg} / \mathrm{kg} /$ day of $1 \alpha-(\mathrm{OH})$ $\mathrm{D}_{3}$ was administered intraperitoneally to male S.D. rats during the experiment. Immediately after or 5 days after the first $1-\alpha-(\mathrm{OH}) \mathrm{D}_{3}$ administration, CS-ALN-NP patches (TP3) were applied on dehaired abdomen skin of the rats for 24 hours at a dose of $0.4 \mathrm{mg}$ and 1.6 $\mathrm{mg}$ of alendronate $/ \mathrm{kg}$. Separately, $1.6 \mathrm{mg} / \mathrm{kg}$ alendronate was administered orally to the rats. At predetermined time points, under ether anesthesia blood was withdrawn from the cervical vein of the rats. To prevent coagulation anticoagulant heparin sulfate was used. The plasma was separated from the other components of the blood by centrifugation. Calcium concentrations in the plasma were determined by Wako Calcium E test (Wako Pure Chemical Industries, Osaka, Japan).

\section{RESULTS AND DISCUSSION}

\section{Characterization of nanoparticles}

Chitosan-alendronate nanoparticles were prepared using the ionotropic external gelation method.

In this study, chitosan and tripolyphosphate (TPP) were used to prepare the alendronate-loaded nanoparticles. The nanoparticles properties were reported in $\mathrm{Ta}-$ ble 3 .

\section{1) Encapsulation efficiency}

The encapsulation efficiency (E.E) od the formulated nanoparticles significantly depended on the preparation methods and was found to be high. The formulation showing the minimal particle size of $250 \mathrm{~nm}$ showed a good encapsulation efficiency of $99.54 \%$. The strong affinity between chitosan and TPP may be the main reason for the high encapsulation efficiency. Chitosan is a cationic polymer which binds readily with TPP-like crosslinking anionic polymers. Alendronate gets attached to either of the polymers and thus is entrapped efficiently. The encapsulation efficiency increased proportionally to the amount of polymer taken for encapsulation.

\section{2) Particle size, PDI (Polydispersity index)}

The particle size of the formed chitosan-alendronate nanoparticles was characterized using a laser diffraction particle size analyzer. Chitosan-alendronate nanoparticles of various size were formed. Among all the formulations, formulation F5 showed the desired size of $250 \mathrm{~nm}$ and PDI of 0.22 (Table 3). The particle size of nanoparticles was greatly influenced by the concentration of chitosan as well as TPP. The higher the concentration of chitosan, the bigger the size of the formed nanoparticles.
Table 4. In vitro drug release profile of CS-ALN-NP.

\begin{tabular}{lllllll}
\hline Time (hr) & F1 & F2 & F3 & F4 & F5 \\
\hline 0 & 0 & 0 & 0 & 0 & 0 \\
\hline 2 & 35.9 & 28.9 & 20.21 & 18.2 & 15.54 \\
\hline 4 & 70.42 & 56.2 & 35.9 & 29.21 & 25.09 \\
\hline 6 & 82.18 & 78.45 & 55.11 & 45.87 & 37.11 \\
\hline 8 & 88.9 & 84.11 & 78.24 & 65.11 & 59.34 \\
\hline 10 & 97.2 & 92.11 & 84.11 & 78.3 & 72.39 \\
\hdashline 12 & 100 & 97.98 & 92.18 & 89.2 & 82.7 \\
\hline
\end{tabular}

The close spatial distance between the molecules of chitosan at higher concentrations attributed to the increased nanoparticles size.

\section{3) Zeta potential}

Zeta potential of chitosan-alendronate nanoparticles was determined by dynamic light scattering (DLS) and it was found to be 28 which indicated a moderate degree of stability due to inner particle repulsion. This result indicated that the transport and adhesion properties of the chitosan-alendronate nanoparticles can be facilitated by electrostatic attraction between the negatively charged nanoparticles and the positively charged cell membranes.

\section{4) In vitro drug release}

The solvent compatibility, nature of the polymer and the degree of cross-linking determine the release of drug from a polymer network. However, the drug release behavior in the case of ionic networks depends upon ionic interactions or ion exchange, and mass transfer (Mccarthy et al., 2002). The chitosan-alendronate nanoparticles formulations were characterized by the drug release profiles F5 formulation showed nearly $82.7 \%$ release over the period of 12 hours (Table 4). This indicated a sustained release of the drug. Encapsulated chitosan-alendronate colloidal formulation reduces the adverse effects in comparison to the alendronate administered orally.

\section{5) SEM}

The morphology of CS-ALN-NP (F5 formulation) was determined by optical microscopy and SEM and the results were shown in Fig. 1. As the image shows, the particles were spherical with a smooth surface and no aggregation was detected.

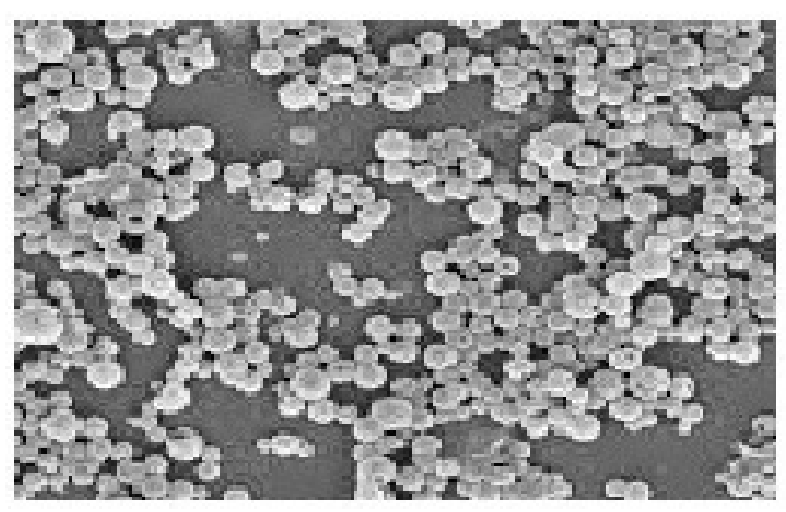

Figure 1. Scanning electron microscopy image of CS-ALN-NP. 
Table 5. Physical characterization of the transdermal patches

\begin{tabular}{cllllllll}
\hline Batch & Appearance & $\begin{array}{l}\text { Thickness } \\
(\mathrm{mm})\end{array}$ & $\begin{array}{l}\text { Wt variation } \\
(\mathrm{g})\end{array}$ & $\begin{array}{l}\text { Folding en- } \\
\text { durance }\end{array}$ & $\begin{array}{l}\text { Drug content } \\
\text { uniformity } \\
(\%)\end{array}$ & $\begin{array}{l}\text { Tensile strength } \\
\left(\mathrm{kg} / \mathrm{cm}^{2}\right)\end{array}$ & $\begin{array}{l}\% \text { Moisture } \\
\text { absorption }\end{array}$ & $\begin{array}{l}\% \text { Moisture } \\
\text { loss }\end{array}$ \\
\hline TP1 & $\mathrm{T}$ & $0.12 \pm 0.03$ & $0.13 \pm 2$ & 138 & $95.20 \pm 1.0$ & $0.26 \pm 0.04$ & $1.7 \pm 0.15$ & $3.5 \pm 1$ \\
\hline TP2 & $\mathrm{S}$ & $0.12 \pm 0.01$ & $0.12 \pm 2$ & 150 & $96.37 \pm 1.5$ & 0.280 .03 & $1.6 \pm 0.20$ & $2.5 \pm 2$ \\
\hdashline TP3 & ST & $0.12 \pm 0.02$ & $0.15 \pm 0.5$ & 165 & $99.66 \pm 0.9$ & $0.32 \pm 0.02$ & $2.5 \pm 0.21$ & $1 \pm 1$ \\
\hdashline TP4 & R & $0.15 \pm 0.03$ & $0.17 \pm 2$ & 200 & $92.30 \pm 1.1$ & $0.38 \pm 0.03$ & $2.5 \pm 0.2$ & $0.5 \pm 1$ \\
\hdashline TP5 & HR & $0.18 \pm 0.02$ & $0.18 \pm 1$ & 220 & $93.36 \pm 1.0$ & $0.50 \pm 0.01$ & $1.7 \pm 0.2$ & $0.2 \pm 1.5$ \\
\hline
\end{tabular}

T, Transparent; S, Smooth, ST, Smooth transparent; R, Rough; HR, Hard and rough

Table 6. Comparison of alendronate permeation through the rat skin.

\begin{tabular}{llllll}
\hline Time (hr) & TP1 & TP2 & TP3 & TP4 & TP5 \\
\hline 0 & 0 & 0 & 0 & 0 & 0 \\
\hline 2 & 12.4 & 10.14 & 9.11 & 7.12 & 6.59 \\
\hline 4 & 33.5 & 30.12 & 25.18 & 22.9 & 18.24 \\
\hline 6 & 45.9 & 43.11 & 40.21 & 35.14 & 30.12 \\
\hline 8 & 55.45 & 53.11 & 46.34 & 41.25 & 35.56 \\
\hline 10 & 67.18 & 65.22 & 60.18 & 55.18 & 46.1 \\
\hline 12 & 78.7 & 72.11 & 69.44 & 62.8 & 55.4 \\
\hline
\end{tabular}

\section{Characterization of the transdermal patches containing CS-ALN-NP}

The transdermal patches containing CS-ALN-NP from optimized formulation F5 were prepared by the solvent casting technique and were characterized for thickness, weight variation, folding endurance, drug content uniformity, tensile strength, percent moisture absorption, percent moisture loss, in vitro drug release and in hypocalcaemia experiment.

\section{Physical appearance}

All the films were evaluated for their physical parameters (weight, thickness, folding endurance, diameter, area, flatness, and surface $\mathrm{pH}$ ), and they were found to be flexible, uniform, smooth, and transparent (Table 5). All the formulations were uniform in their weight, thickness, folding endurance, and diameter, with low S.D. values. The thickness of the patches varied from $0.12 \pm 0.01 \mathrm{~mm}$ to $0.18 \pm 0.02 \mathrm{~mm}$. In the film thickness measurements, low standard deviation values proved the uniformity of the patches. The weight of the prepared transdermal patches ranged between $0.12 \pm 2 \mathrm{~g}$ and $0.18 \pm 1 \mathrm{~g}$. Folding endurance values varied between 138 and 220 . The drug content uniformity ranged from $92.30 \pm 1.1$ to $99.66 \pm 0.9$. The tensile strength ranged from $0.26 \pm 0.04$ to $0.50 \pm 0.01$. The percentage moisture absorption ranged from $1.6 \pm 0.20$ to $2.5 \pm 0.21$. The percentage moisture loss ranged from $0.2 \pm 1.5$ to $3.5 \pm 1$.

\section{In vitro skin permeation study}

Patches containing CS-ALN-NP were subjected to in vitro rat abdominal skin permeation study. The patch (all formulations TP1 to TP5) was applied to the stratum corneum side of the separated skin. The receptor compartment of the cell was filled with $3 \mathrm{ml}$ of phosphate buffer solution $\mathrm{pH}$ 7.4. The transdermal permeation of alendronate over 12 hours was found to be between

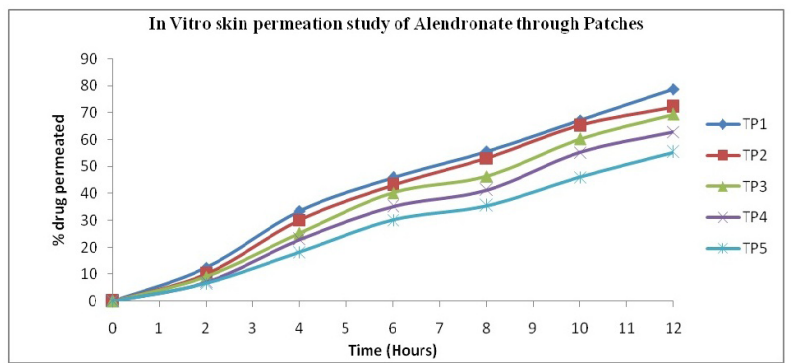

Figure 2. In vitro skin permeation study of alendronate from the patches

$55.4 \%$ and $72.11 \%$. The comparison of the permeation values was shown in Table 6 and presented in Fig. 2. The TP3 formulation had the drug content of $99.96 \%$ which was the highest among all of the formulations and showed relatively controlled skin permeation of $69.44 \%$ over the period of 12 hours and was considered the optimized formulation. TP3 formulation was further used for the animal study in order to determine the calcium level and plasma concentration of alendronate.

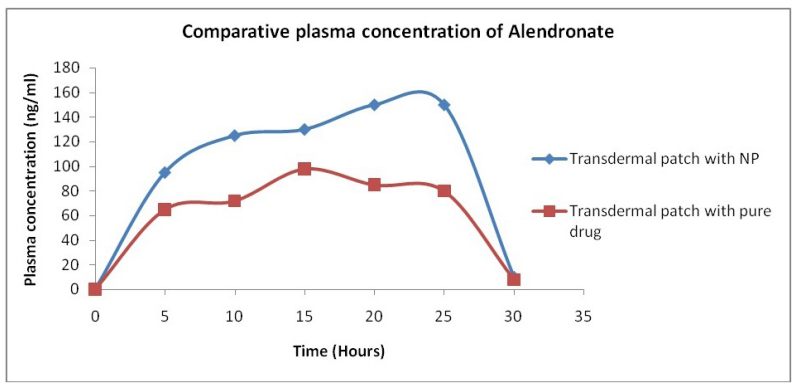

Figure 3. Comparison of the plasma concentration of alendronate. 
Table 7. Pharmacokinetic parameters of alendronate.

\begin{tabular}{lll}
\hline Parameters & $\begin{array}{l}\text { Transdermal patch } \\
\text { with pure drug }\end{array}$ & $\begin{array}{l}\text { Transdermal patch } \\
\text { with NP }\end{array}$ \\
\hline Dose $(\mathrm{mg} / \mathrm{kg})$ & 30 & 10 \\
\hline AUC $(\mathrm{ng} . \mathrm{h} / \mathrm{mL})$ & 905 & 98 \\
\hline BA (\%) & 1.2 & 7.4 \\
\hline
\end{tabular}

Table 8. Comparative plasma concentration

\begin{tabular}{|c|c|c|}
\hline \multirow{2}{*}{ Days } & \multicolumn{2}{|c|}{ Plasma calcium concentration (mg/dl) } \\
\hline & $\begin{array}{l}\text { Transdermal patch with } \\
\text { pure drug }\end{array}$ & $\begin{array}{l}\text { Transdermal patch with } \\
\text { CS-ALN-NP }\end{array}$ \\
\hline 0 & 16 & 16 \\
\hline 1 & 16 & 15 \\
\hline 2 & 16 & 12 \\
\hline 3 & 15 & 11 \\
\hline 4 & 14.5 & 9 \\
\hline 5 & 13 & 8 \\
\hline 6 & 13 & 8 \\
\hline 7 & 12 & 4 \\
\hline
\end{tabular}

Plasma concentration of alendronate

Figure 3 shows the comparison of plasma concentration of alendronate when administered as pure drug and as nanoparticles. It could be observed that alendronate rapidly disappeared from the blood circulation after it was used as a pure drug in the transdermal patches. The bioavailability was also found very low $(1.2 \%$ at a dose of $30 \mathrm{mg} / \mathrm{kg})$. In contrast, the plasma concentration of alendronate increased gradually during the first five hours at a dose of $10 \mathrm{mg} / \mathrm{gg}$ when CS-AL-NP was used in the patches. The plasma concentration was maintained at a constant level over a period of 25 hours. Also, the bioavailability of this formulation was found to be $7.4 \%$ at a dose of $10 \mathrm{mg} / \mathrm{kg}$. In other words, nearly six-time enhancement of bioavailability was observed when alendronate was used in the transdermal patches in the nanoparticulate form. Pharmacokinetic parameters were shown in Table 7.

\section{Hypercalcemia experiment}

Figure 4 shows the plasma calcium concentration profile in hypercalcemia model rats treated with two different transdermal patch formulations. It could be observed

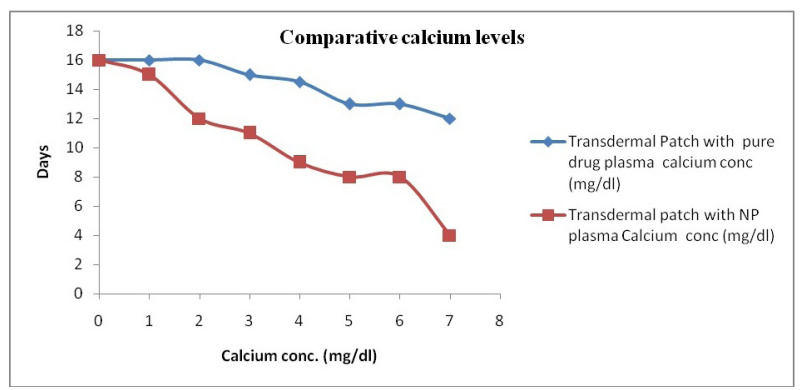

Figure 4. Plasma calcium concentration profile in hypercalcemia model rats treated with two different transdermal patch formulations that the increase in plasma calcium level was almost completely suppressed in rats treated with transdermal patches containing CS-ALN-NP while patches containing pure alendronate did not show a significant decrease in the plasma calcium level. Over the period of seven days, the plasma calcium concentration was decreased from 16 $\mathrm{mg} / \mathrm{dl}$ to $4 \mathrm{mg} / \mathrm{dl}$ (4 times) in rat groups treated with transdermal patches with CS-ALN-NP while the calcium level was as high as $12 \mathrm{mg} / \mathrm{dl}$ in case of transdermal patches containing pure alendronate (refer Table 8).

Alendronate is the first choice drug for the treatment of hypercalcemia in malignancy and osteoporosis (Sato et al., 1990). Our study proved that CS-ALN-NP delivered transdermally had a higher effect on plasma calcium level than pure alendronate and also the bioavailability was found higher as compared to the pure alendronate. These results (enhanced skin permeation, enhanced bioavailability and decrease of calcium level) strongly suggest that transdermal patches with CS-AL-NP inhibit the function of osteoclasts which mediates the bone resorption and leads to preventive and therapeutic effects in the treatment of osteoporosis and hypercalcemia (Sato et al., 1990).

\section{CONCLUSION:}

We successfully developed ab ultrasound-assisted transdermal patch delivery system containing CS-ALNNP for the treatment of osteoporosis. In our study, the skin permeation of alendronate after ultrasound treatment through patch system was found sufficient for the treatment of osteoporosis in the rat model. Also, the bioavailability of the optimized formulation was six times higher as compared to the patch containing pure alendronate. The plasma calcium concentration suppression potential was found significant which would help in the prevention of osteoporosis. Our findings (enhanced skin permeation, enhanced bioavailability and suppression of plasma calcium level) regarding the transdermal delivery system suggest it as a promising approach for the treatment of osteoporosis.

\section{REFERENCES}

Allemann E, Gurny R, Deolker E (1993) Drug loaded nanoparticles: preparation methods and drug targeting issues. Eur. J. Pharm. Biopharm. 39: 173-191.

Dolatabadi EN, Hamishehkar H, Eskandani M. Valizadeh H (2014) Formulation, characterization and cytotoxicity studies of alendronate sodium loaded solid lipid nanoparticles. Colloids Surf. B. Biointerfaces. 1: 21-28. https://doi.org/10.1016/j.colsurfb.2014.01.055

Drake MT, Clarke BL, Khosla S (2008) Bisphosphonates: mechanism of action and role in clinical practice. Mayo Clin. Proc. 83: 10321045. https://doi.org/10.4065/83.9.1032

Dunford JE, Rogers MJ, Ebetino FH (2006) Inhibition of protein prenylation by bisphosphonates causes sustained activation of Rac, Cdc42, and Rho GTPases. J. Bone Miner. Res. 21: 684-694. https:// doi.org/10.1359/jbmr.060118

Ezra A, Golomb G (2000) Administration routes and delivery systems of bisphosphonates for the treatment of bone resorption. Adv. Drug Deliv. Rev. 42: 175-195. https://doi.org/10.1016/s0169409x $(00) 00061-2$

Fisher JE, Rodan GA, Reszka AA (2000) In vivo effects of bisphosphonates on the osteoclast mevalonate pathway. Endocrinology 141: 47934796. https://doi.org/10.1210/endo.141.12.7921

Fleisch H (1987) Experimental basis for the use of bisphosphonates in Paget's disease of bone. Clin. Orthop. Relat. Res. 217: 72-78.

Fleisch H (2002) Development of bisphosphonates. Breast Cancer Res. 4: 30-34. https://doi.org/10.1186/bcr414

Haddad PT, Salazar M, Hernades L (1998) Histomorphometry of the organic matrix of the femur in ovarictomised rats treated with sodum Alendronate. Rev. Bras. Ortop. 50: 100-104. https://doi. org/10.1016/j.rboe.2014.12.007 
Johnell O, Kanis JA (2006) An estimate of the worldwide prevalence and disability associated with osteoporotic fractures. Osteoporos Int. 17: 1726-1733. https://doi.org/10.1007/s00198-006-0172-4

Kaveh K, Ibrahim R, Abubakar M, Ibrahim TA (2010) Osteoporosis induction in animal model. J. Anim. Vet. Adv. 9: 1055-1067. https://doi.org/10.3844/ajavsp.2010.139.145

Lambrinoudaki I, Christodoulakos G, Botsis D (2006) Bisphosphonates. Ann. NY. Acad. Sci. 1092: 397-402. https://doi.org/10.1196/ annals.1365.036

Mccarthy EA, Raggio CL, Hossack MD, Miller EA, Jain S, Boskey AL, Camacho NP (2002) Alendronated treatment for infants with osteogenesis Imperfecta: Demonstrationof Efficacy in Mouse Model. Pediatric Res. 52: 660-670. https://doi.org/10.1203/00006450200211000-00010

Morimoto Y, Mutoh M, Ueda H, Fang L, Hirayama K, Atobe M, Kobayashi D (2005) Elucidation of the transport pathway in hairless rat skin enhanced by lowfrequency sonophoresis based on the solutewater transport relationship and confocal microscopy. J. Control. Release 103: 587-597. https://doi.org/10.1016/j.jconrel.2005.01.005

Paliwal S, Menon GK, Mitragotri S (2006) Low-frequency sonophoresis: ultrastructural basis for stratum corneum permeability assessed using quantum dots. J. Invest. Dermatol. 126: 1095-1101. https://doi. org/10.1038/sj.jid.5700248

Reid IR (1997) Glucocorticoid osteoporosis-mechanism and management. Eur. J. Endocrinol. 137: 209-217.

Reszka AA, Rodan GA (2003) Bisphosphonate mechanism of action. Curr Rheumatol Rep. 5: 65-74. https://doi.org/10.1530/eje.0.1370209
Rodan GA, Reszka AA (2002) Bisphosphonate mechanism of action. Curr. Mol. Med. 2: 571-577. https://doi. org/10.2174/1566524023362104

Russell RG (2007) Bisphosphonates: mode of action and pharmacology. Pediatrics 119 (Suppl 2): S150-S162. https://doi.org/10.1542/ peds.2006-2023H

Sato M, Grasser W (1990) Effects of bisphosphonates on isolated rat osteoclasts as examined by reflected light microscopy. J. Bone Miner Res. 5: 31-40. https://doi.org/10.1002/jbmr.5650050107

Saunders Y, Ross JR, Broadley KE (2004) Steering Group Systematic review of bisphosphonates for hypercalcaemia of malignancy. Palliat Med. 18: 418-431. https://doi.org/10.1191/0269216304pm914ra

Schenk R, Eggli P, Fleisch H (1986) Quantitative morphometric evaluation of the inhibitory activity of new aminobisphosphonates on bone resorption in the rat. Calcif Tissue Int. 38: 342-349. https://doi. org/10.1007/BF02555748

Sözen T, Ozışık L, BaşaranBaşaran NÇ, Tumay S, Lale Ozışık, Çalık BN (2017) An overview and management of osteoporosis. Eur. J. Rheumatol. 4: 46-56. https://doi.org/10.5152/eurjrheum.2016.048

VanBeek ER, Cohen LH, Leroy IM (2003) Differentiating the mechanisms of antiresorptive action of nitrogen containing bisphosphonates. Bone 33: 805-811. https://doi.org/10.1016/j.bone.2003.07.007

Wen LD, Shan SN, Ying LX, Zi RX, Cheng LF (2009) Antibacterial activity of chitosan tripolyphosphate nanoparticles loaded with various metal ions. Carbohydrate Polymers 75: 385-389. https://doi. org/10.1016/j.carbpol.2008.07.039 